\title{
No evidence for DNA copy number change associated with the DUP25 cytogenetic phenotype
}

\author{
European Journal of Human Genetics (2003) 11, 911-912. doi:10.1038/sj.ejhg.5201074 \\ Published online 24 September 2003
}

A cytogenetic variant at chromosome 15q24-26 associated with anxiety disorder has been previously identified. ${ }^{1}$ It showed a number of interesting features, including somatic and germline mosiacism, non-linkage to neighbouring genetic markers and non-Mendelian inheritance. However, a recent report from a laboratory in Salisbury has failed to replicate these findings, and did not demonstrate this cytogenetic variant among 16 patients and 40 controls, including three previously verified as DUP25 positive by Gratacos and co-workers in Barcelona. ${ }^{2}$ Another study using multicolor FISH has also failed to confirm the presence of DUP25. ${ }^{3}$

In light of this difference, and of the potential clinical importance of DUP25, we have developed a direct assay for DNA dosage at three loci within the DUP25 region using multiplex amplifiable probe hybridisation (MAPH). ${ }^{4} \mathrm{MAPH}$ has been used to reliably detect dosage at several loci, including DMD and subtelomeric regions of chromosomes. ${ }^{5,6}$ The three MAPH probes made for DUP25 analysis map to three sequence-tagged sites that were used to verify clone assembly in the original DUP25 investigation. They were WI-7231 (DDBJ/EMBL/Genbank accession number G06467, final exon of CRABP1), WI-7494 (accession number U30227, intron 6 of NTRK3) and WI-1407 (accession number G05361, intron 4 of RPS17). The probe
WI-7494 maps to NTRK3, which, by using two DNA-based dosage methods (Southern blot quantification and quantitative-PCR), was used to study DUP25 dosage in the original investigation. ${ }^{1}$ These three probes were integrated into a MAPH subtelomeric probe screening set, ${ }^{6}$ which acted as a reference framework in which each probe shows a copy number of 2 per diploid cell.

We analysed 12 samples that had been typed cytogenetically for DUP25, 15 normal controls and one trisomy 15 positive control (Table 1). The values are normalised to control samples so that the normal diploid dosage will be reported by a value of 1.00 , with heterozygous duplication reported as 1.5. Owing to the mosaic nature of DUP25, values between 1.0 and 1.5 would reflect the mosaic nature of the DNA preparation. For example, DNA derived from a patient with 50\% somatic mosaicism would have an expected value of 1.25 . The values shown are mean of several replicates $\pm 95 \%$ confidence intervals; multiple measurements were made in order to minimise experimental noise and maximise mosaicism detection sensitivity. However, at low \%DUP25 mosaicism, the power to detect any duplication by MAPH weakens.

Table 1 shows that all three probes detect a duplication (copy number of 3 ) for the trisomy 15 positive control, and reported diploid dosage of 2 for all normal controls.

Table 1 Analysis of DUP25 samples by MAPH

\begin{tabular}{|c|c|c|c|c|c|c|}
\hline Sample & Source & $\% D U P 25$ & $n$ & Wl-7231 mean $\pm 95 \% \mathrm{Cl}$ & WI-7494 mean $\pm 95 \% \mathrm{Cl}$ & WI-1407 mean $\pm 95 \% \mathrm{Cl}$ \\
\hline P5 & $\mathrm{L}$ & 100 & 5 & $1.19 \pm 0.06$ & $1.01 \pm 0.05$ & $1.07 \pm 0.08$ \\
\hline PH188 & B & 90 & 1 & $1 . \overline{15}$ & 1.24 & 1.15 \\
\hline PHE6 & B & 90 & 3 & $1.01 \pm 0.06$ & $1.00 \pm 0.08$ & $1.07 \pm 0.06$ \\
\hline P3 & $\mathrm{L}$ & 80 & 5 & $1.10 \pm 0.12$ & $1.01 \pm 0.04$ & $0.98 \pm 0.07$ \\
\hline PHE35 & B & 80 & 3 & $1.04 \pm 0.07$ & $0.97 \pm 0.10$ & $0.99 \pm 0.04$ \\
\hline W2 & $\mathrm{L}$ & 45 & 2 & $1.13 \pm 0.24$ & $1.08 \pm 0.03$ & $1.12 \pm 0.06$ \\
\hline W4 & $\mathrm{L}$ & 40 & 2 & $1.00 \pm 0.17$ & $1.08 \pm 0.21$ & $1.24 \pm 0.05$ \\
\hline PH6 & $\mathrm{L}$ & 36 & 5 & $1.37 \pm 0.10$ & $1.01 \pm 0.18$ & $1.12 \pm 0.12$ \\
\hline D6 & B & $<20$ & 3 & $0.96 \pm 0.02$ & $0.93 \pm 0.03$ & $0.99 \pm 0.11$ \\
\hline X75 & B & $<20$ & 2 & $0.90 \pm 0.02$ & $0.94 \pm 0.04$ & $0.85 \pm 0.14$ \\
\hline PH122 & $\mathrm{L}$ & 18 & 5 & $1.11 \pm 0.05$ & $1.02 \pm 0.11$ & $1.20 \pm 0.10$ \\
\hline PH67 & $\mathrm{L}$ & 17 & 5 & $1.06 \pm 0.06$ & $1.12 \pm 0.08$ & $1.05 \pm 0.11$ \\
\hline W1 & L & Trisomy 15 & 2 & $1.54 \pm 0.07$ & $1.57 \pm 0.24$ & $1.61 \pm 0.03$ \\
\hline
\end{tabular}

All samples normalised to 14 controls (normal diploid dosage mean $=1, S D=0.09$ ), except $\mathrm{W}$ samples which are normalised to a cytogenetically verified normal cell line (mean $=1.00, S D=0.1) . n=$ times tested; $L=$ lymphoblast cell line; $B=$ fresh peripheral blood. \%DUP25 is the percentage of DUP25 cytogenetic variant in the sample, as measured by the Barcelona laboratory (L Armengol, $X$ Estivill, personal communication). 
However, the probes failed to detect any consistent copy number change over the three probes in individuals assayed for DUP25 by cytogenetic analysis. For two patients, W2 and W4, DUP25 had been detected by the Barcelona laboratory, but could not be detected by the Salisbury laboratory. ${ }^{2}$ Only one of the three test results that show values greater or equal to 1.2 in repeated tests (W4, WI-1407; PH6, WI-7231; PH122, WI-1407) is significantly different from either of the values reported by the other two probes for that sample (PH6, WI-7231, value of 1.37; one tailed $t$-test, null hypothesis of equal means, $P<0.05$, not corrected for multiple tests). Although further work would be needed to confirm it, this probe may be reporting a small duplication that is not detected by the other probes, but which is clearly unrelated to DUP25.

In summary, our evidence from MAPH analysis fails to confirm any DNA copy number change at three loci mapping to the DUP25 region at 15q24-26 in patients previously shown by cytogenetics to have DUP25. There are two possible reasons for this discrepancy: either the DUP25 cytogenetic change does not involve a corresponding alteration of DNA copy number, or the cytogenetic appearance reported as DUP25 is not a consistent change in chromosome structure, but is an experimental artefact.

\section{Acknowledgements}

We acknowledge Xavier Estivill, Lluis Armengol, Monica Gratacos, John Crolla and Patricia Jacobs for samples, and Ingrid Davies for ABI 377 gel running service. This work was funded by Wellcome Trust Grant number 060578 to JALA.
Edward J Hollox and John AL Armour Institute of Genetics, University of Nottingham, School of Medicine, Queen's Medical Centre, Nottingham, UK

Correspondence: EJ Hollox, Institute of Genetics, University of Nottingham, School of Medicine, Queen's Medical Centre, Nottingham NG7 2UH, UK.

Tel.: + 44-115-924-9924 x 43233; Fax: + 44-115-970-9906;

E-mail: ed.hollox@nottingham.ac.uk

\section{References}

1 Gratacos M, Nadal M, Martin-Santos R et al: A polymorphic genomic duplication on human chromosome 15 is a susceptibility factor for panic and phobic disorders. Cell 2001; 106: $367-379$.

2 Tabiner M, Youings S, Dennis N et al: Failure to find DUP25 in patients with anxiety disorders, in control individuals, or in previously reported positive control cell lines. Am J Hum Genet 2003; 72: 535-538.

3 Weiland Y, Kraus J, Speicher M: A multicolor FISH assay does not detect DUP25 in control individuals or in reported positive control cells. Am J Hum Genet 2003; 72: 1349-1352.

4 Armour JA, Sismani C, Patsalis PC, Cross G: Measurement of locus copy number by hybridisation with amplifiable probes. Nucleic Acids Res 2000; 28: 605-609.

5 White S, Kalf M, Liu Q et al: Comprehensive detection of genomic duplications and deletions in the DMD gene, by use of multiplex amplifiable probe hybridization. Am J Hum Genet 2002; 71: $365-374$.

6 Hollox EJ, Atia T, Cross G, Parkin T, Armour JAL: High-throughput screening of human subtelomeric DNA for copy number changes using multiplex amplifiable probe hybridisation (MAPH). J Med Genet 2002; 39: 790-795. 